\title{
Independent Risk Factors and The Long-term Outcomes for Postoperative Continuous Renal Replacement Treatment in Patients Who Underwent Emergency Surgery for Type A Acute Aortic Dissection
}

\section{Zhigang Wang}

Nanjing University Medical School Affiliated Nanjing Drum Tower Hospital

Min Ge

Nanjing University Medical School Affiliated Nanjing Drum Tower Hospital

\section{Tao Chen}

Nanjing University Medical School Affiliated Nanjing Drum Tower Hospital

Cheng Chen

Nanjing University Medical School Affiliated Nanjing Drum Tower Hospital

Qiuyan Zong

Nanjing University Medical School Affiliated Nanjing Drum Tower Hospital

Lichong Lu

Nanjing University Medical School Affiliated Nanjing Drum Tower Hospital

Dongjin Wang ( $D$ glyywdj@163.com )

Nanjing University Medical School Affiliated Nanjing Drum Tower Hospital https://orcid.org/00000003-3187-7916

\section{Research article}

Keywords: Aortic dissection, Continuous renal replacement therapy, Outcomes, Serum creatinine, Cardiopulmonary bypass, Thoracic aortic surgery

Posted Date: May 20th, 2020

DOl: https://doi.org/10.21203/rs.3.rs-15942/v2

License: (c) (i) This work is licensed under a Creative Commons Attribution 4.0 International License.

Read Full License 
Version of Record: A version of this preprint was published at Journal of Cardiothoracic Surgery on May 15th, 2020. See the published version at https://doi.org/10.1186/s13019-020-01153-8. 


\section{Abstract}

Objective: The study objective was to investigate the incidence and risk factors of continuous renal replacement therapy (CRRT) in patients undergoing emergency surgery for type A acute aortic dissection (TA-AAD) and evaluate the perioperative and long-term outcomes.

Methods: From January 2014 to December 2018, 712 consecutive patients were enrolled in the study. These patients were divided into two groups according to whether or not needed postoperative CRRT: the CRRT group vs the control group. Univariate analysis and binary logistic regression analysis were used to analyze the risk factors of CRRT. To avoid the selection bias and confounders, baseline characteristics were matched for propensity scores. Kaplan-Meier curves were generated to provide survival estimates at postoperative points in time.

Results: Before propensity score matching, univariate analysis showed that there were significant differences in age, preoperative hypertension, pericardial effusion, preoperative serum creatinine $(\mathrm{sCr})$, intraoperative need for combined coronary artery bypass grafting (CABG) or mitral valve or tricuspid valve surgery, cardiopulmonary bypass (CPB) time, extracorporeal circulation assistant time, aortic cross-clamp time, drainage volume 24 hours after surgery and ventilator time between two groups. All were higher in the CRRT group $(P<0.05)$. These risk factors were included in binary logistic regression. It showed that preoperative $\mathrm{SCr}$ and $\mathrm{CPB}$ time were independent risk factors for CRRT patients undergoing surgery for TA-AAD. And there were significant differences regarding 30-day mortality $(P<0.001)$ and long-term overall cumulative survival $(P<0.001)$ with up to a 6-year follow-up. After propensity scoring, 29 pairs (58 patients) were successfully matched. Among these patients, the analysis showed that CPB time was still significantly longer in the CRRT group $(P=0.004)$, and the 30 -day mortality rate was also higher in this group $(44.8 \%$ vs $10.3 \%$; $=0.003)$.

Conclusion: CRRT after TA-AAD is common and worsened short- and long- term mortality. The preoperative $\mathrm{s} C r$ and $\mathrm{CPB}$ time are independent risk factors for postoperative CRRT patients. Shorten the CPB time as much as possible is recommended to reduce the risk of CRRT after the operation.

\section{Introduction}

Previous studies have shown a high occurrence of acute kidney injury (AKI) in patients undergoing cardiothoracic surgery procedures. It is related to increased mortality, morbidity, longer intensive care unit (ICU) stay time and reduces late survival [1-4]. Continuous renal replacement therapy (CRRT) is an effective treatment strategy for those patients with acute renal failure (ARF), especially those who require both circulatory and respiratory support.

Although mild to moderate acute kidney injuries has a high incidence [5], 2-15\% of patients with AKI need CRRT after aortic surgery, and especially higher in those with TA-AAD surgery $[1,6,7]$. Despite continued progress in renal replacement therapy and intensive care in the past years, the short- and longterm mortality still remains high, with a rate ranging from $50 \%$ to over $80 \%$ in patients undergoing CRRT 
[8]. However, there is scarce literature on the incidence, risk factors and long-term outcomes for CRRT in patients with TA-AAD repair. Identification of risk factors for CRRT in patients with TA-AAD may lead to timely initiation of CRRT, and improve clinical outcomes. This retrospective study aims to identify the risk factors and 30-day and long-term outcomes for CRRT after surgical repair in patients with TA-AAD.

\section{Methods And Materials}

A total of 730 consecutive patients with TA-AAD in our hospital from January 2014 to December 2018 were retrospectively analyzed. Previous approval was obtained from the institutional research ethics committee, which waived the need for individual informed consent. Aortic dissection was diagnosed by computed tomography angiography at either our institution or the referring hospital. Patients with a history of chronic renal failure (CRF) or with intraoperative and postoperative death within 24 hours were excluded. Patients were divided into the control group (601 cases) and the CRRT group (111 cases) according to whether they received CRRT after surgery. The basic clinical data of the two groups are shown in table 1.

Demographic variables included age, gender, body mass index (BMI), previous medical history (hypertension, diabetes, cardiac surgery, coronary artery disease, cerebrovascular disease), aortic dissection features (blood supply of renal artery) and pericardial effusion. Operation-related variables were the duration of cardiopulmonary bypass (CPB) and aortic cross-clamping, extracorporeal circulation assist, the duration of deep hypothermic circulatory arrest (DHCA). Laboratory variables included preoperative serum creatinine ( $\mathrm{sCr}$ ) and serum blood urea nitrogen (BUN) levels. Postoperative variables included drainage volume 24 hours after surgery, duration of mechanical ventilation, ICU and hospital stay, and 30-day mortality.

Criteria for the initiation and termination of CRRT after severe AKI is referred to the guidelines for the clinical practice of AKI from the global organization for the improvement of renal prognosis: Kidney Disease Improving Global Outcomes (KDIGO) [9]. CRRT was considered in patients with the increase of sCr more than $26.5 \mathrm{umol} / \mathrm{L}$ within 48 hours after surgery or the urine volume was less than $0.5 \mathrm{ml} / \mathrm{kg} / \mathrm{h}$ lasting for $6 \mathrm{~h}$, and serum $\mathrm{K}^{+}>6.0 \mathrm{mmol} / \mathrm{L}$ or $\mathrm{HCO}_{3}<10 \mathrm{mmol} / \mathrm{L}$. Within $48 \mathrm{~h}$ after the last CRRT, if $\mathrm{sCr}$ decreased $>50 \mathrm{umol} / \mathrm{L}$ (the sampling interval greater than $12 \mathrm{~h}$ ) or urine volume $>0.5 \mathrm{ml} / \mathrm{kg} / \mathrm{h}$ within $12 \mathrm{~h}$, serum $\mathrm{K}^{+}<5.5 \mathrm{mmol} / \mathrm{L}$ and $\mathrm{HCO}_{3}{ }^{-}>18 \mathrm{mmol} / \mathrm{L}, \mathrm{CRRT}$ was considered for termination, as introduced in previous studies [10].

CRRT was performed in our department, using the $11.5 \mathrm{f}$ double-chamber dialysis catheter, the AV600S polysulfone membrane blood filter and the connection pipeline of blood filtration, infusion pump, and syringe pump. The internal jugular vein or femoral vein or subclavian vein was selected to place a single double-chamber blood filter catheter. The hemodynamic force is provided by the blood pump. $1000 \mathrm{ml}$ heparin brine was pre-flushed before using the filter to empty the air bubbles in the filter and pipeline. We then placed the sterile collecting bag $30-50 \mathrm{~cm}$ below the filter, and recorded the flow of liquid in and out every hour. In the early postoperative patients after aortic dissection, local anticoagulation of prefilter 
citrate was used to reduce bleeding. The replacement solution was $0.9 \%$ normal saline and $5 \%$ glucose solution, with a ratio of $3: 1$. Additionally, $250 \mathrm{ml} 5 \%$ sodium bicarbonate was added for q4h or q6h to timely supplement the physiological needs and nutrients lost by blood filtration. The input method is pre or post-dilution method, which can balance the fluid and adjust the infusion speed according to the amount of filtrate and input. The filter is usually replaced after a blockage or when the filtrate drops, and the continuous veno-venous hemofiltration (CVVH) blood flow should be $100-150 \mathrm{ml} / \mathrm{min}$. To maintain perfusion pressure of kidney, vasopressor and inotropic drugs were routinely used in TA-AAD patients with postoperative hypotension.

\section{Surgical Procedure}

The median sternal incision was used in all surgeries under general anesthesia and DHCA. All patients were treated with Terumo inlet membrane lung, no pre-rinse containing sugar was used in extracorporeal circulation, ultrafiltration and autologous blood recovery devices were routinely used. Extracorporeal circulation was established by a routine femoral artery or right axillary artery and right atrial intubation. When the nasopharyngeal temperature dropped to $34^{\circ} \mathrm{C}$, the ascending aorta was clamped and cardiac arrest fluid was injected to complete the operation of the proximal end of the aorta. When the nasopharyngeal temperature dropped to $18 \sim 20^{\circ} \mathrm{C}$ and the bladder temperature to $22 \sim 24^{\circ} \mathrm{C}$, systemic circulatory was arrested, and the flow was reduced to $3 \sim 5 \mathrm{ml} \cdot \mathrm{kg}^{-1} \cdot \mathrm{min}^{-1}$ to complete the operation of the aortic arch and descending arch. Near-infrared spectroscopy was used to monitor cerebral oxygenation in the bilateral frontal lobes during the DHCA time. All patients returned to the ICU for routine monitoring and treatment.

\section{Statistical Analysis}

SPSS 25.0 software was used for statistical analysis. Univariate analysis was performed for each variable. Data were compared using the Student $t$ test or non- parametric Wilcoxon Mann-Whitney $U$ test for continuous variables and the chi-squared or Fisher's exact test for categorical variables before matching; The multivariate model included variables that were significant on univariate analysis. Baseline characteristics (Demographic variables, previous medical history, aortic dissection features and pericardial effusion) were matched for propensity scores. We performed one-to-one pair matching using nearest neighbor matching without replacement within 0.02 standard deviations of the logit of the propensity score as caliper width.

Kaplan-Meier curves were generated to provide survival estimates at postoperative points in time. Differences between the 2 groups were determined by log-rank tests. For all analyses, a probability value of less than 0.05 was considered statistically significant. 


\section{Results}

Three patients with CRF before surgery and 15 patients who died intraoperatively or within 24 hours after surgery were excluded, a total of 712 patients were included in the study. Among them, 111 cases (15.9\%) underwent CRRT after thoracic aortic surgery. For the CRRT group, there were 84 cases $(75.7 \%)$ of males, the medical history included hypertension in 91 cases $(82 \%)$, diabetes in 1 case $(0.9 \%)$, coronary heart disease in 9 cases $(8.1 \%)$, cardiovascular surgery in 8 cases $(7.2 \%)$, cerebrovascular accident in 4 cases (3.6\%), and pericardial effusion in 13 cases (11.7\%). (Table 1) The operation methods included aortic root or ascending aorta replacement in 49 cases (44.1\%), total aortic arch replacement in 62 cases (55.9\%), and aortic valve treatment in 31 cases (27.9\%), coronary artery bypass grafting (CABG) or mitral or tricuspid valve operation in 17 cases (15.3\%). 107 patients (96.4\%) received emergency operations. The mean CPB duration was $273.4 \pm 80.6$ minutes, the duration of aortic cross-clamp was $186.0 \pm 70.5$ minutes, and the mean duration of DHCA was 30.2 \pm 50.1 minutes, as shown in Table 2 .

Compared with the CRRT group, preoperative age, hypertension, pericardial effusion, preoperative sCr and BUN, intraoperative need for combined CABG or mitral valve or tricuspid valve surgery, CPB time, extracorporeal circulation assistant time, aortic crossclamp time, drainage volume 24 hours after surgery, ventilator time, 30-day mortality, ICU time between two groups $\square$ all above results were higher in CRRT group $(P<0.05)$. There was no significant difference in hospital stay between the two groups. (Table 1,2)

Binary logistic regression analysis showed that preoperative sCr $(O R=1.008,95 \% C I$ : 1.002-1.014, $P=0.005)$ and CPB time ( $O R=1.022,95 \% C I: 1.003-1.042, P=0.026)$ were independent risk factors for CRRT after thoracic aortic surgery. (Table 3)

After propensity scoring, 29 pairs (58 patients) were successfully matched. In these patients, the analysis showed that CPB time was significantly longer in CRRT group $(P=$ $0.004)$, and the 30 -day mortality rate was also higher in this group (44.8\% vs $10.3 \% ; P=$ 0.003). (Table 2)

51 patients in the CRRT group and 43 patients in the control group died during the hospitalization period. At last, a total of 60 CRRT patients and 558 non-CRRT patients were included in the follow-up. The median follow-up time was 29 months. 48 patients were lost from follow-up, including 47 patients in the control group and 1 in the CRRT group. They were excluded from the subsequent long-term survival analysis. There were 41 late deaths, it is important to point out that 1 patient in the control group committed suicide 6 months after discharge and was excluded from the study. Finally, 25 deaths (4.9\%) in the control group and $15(25.4 \%)$ in the CRRT group were identified $(P<0.001)$. The survival is shown in Fig. 1. 
Table 1 Baseline clinical characteristics of patient population and aortic dissection features before and after propensity score matching

\begin{tabular}{|c|c|c|c|c|c|c|}
\hline \multirow[b]{2}{*}{ Characteristics } & \multicolumn{3}{|c|}{ Before Matching } & \multicolumn{3}{|c|}{ After Matching } \\
\hline & $\operatorname{Control}(n=601)$ & $\operatorname{CRRT}(\mathrm{n}=111)$ & $P$ Value & Control $(n=29)$ & $\operatorname{CRRT}(\mathrm{n}=29)$ & $P$ Value \\
\hline \multicolumn{7}{|l|}{ Patient characteristics } \\
\hline \multicolumn{7}{|l|}{ Demographics } \\
\hline Age (year) & $52.0 \pm 13.0$ & $55.4 \pm 14.0$ & 0.011 & $54.6 \pm 13.2$ & $54.8 \pm 13.2$ & 0.937 \\
\hline Male (\%) & $438(72.9)$ & $84(75.7)$ & 0.540 & $20(69.0)$ & $23(79.3)$ & 0.368 \\
\hline BMI $(\mathrm{kg} / \mathrm{m} 2)$ & $25.2 \pm 5.0$ & $26.1 \pm 5.0$ & 0.146 & $25.8 \pm 3.4$ & $27.0 \pm 5.0$ & 0.278 \\
\hline \multicolumn{7}{|l|}{ Medical history } \\
\hline Hypertension (\%) & $401(66.7)$ & $91(82.0)$ & 0.001 & $20(69.0)$ & $21(72.4)$ & 0.773 \\
\hline Diabetes mellitus (\%) & $11(1.8)$ & $1(0.9)$ & 0.703 & 0 & 0 & -- \\
\hline Previous cardiac surgery (\%) & $28(4.7)$ & $8(7.2)$ & 0.260 & 0 & 0 & -- \\
\hline Previous Coronary artery disease (\%) & $18(3.0)$ & $9(8.1)$ & 0.025 & $1(3.4)$ & 2(6.9) & 1.000 \\
\hline Cerebrovascular disease (\%) & $20(3.3)$ & $4(3.6)$ & 0.779 & $2(6.9)$ & $1(3.4)$ & 1.000 \\
\hline Pericardial effusion (\%) & $15(2.5)$ & $13(11.7)$ & $<0.001$ & $1(3.4)$ & $1(3.4)$ & 1.000 \\
\hline \multicolumn{7}{|l|}{ Preoperative laboratory data } \\
\hline BUN (mmol/L) & $7.0 \pm 3.8$ & $11.5 \pm 8.2$ & $<0.001$ & $7.8 \pm 3.0$ & $6.6 \pm 1.9$ & 0.063 \\
\hline $\mathrm{sCr}(\mu \mathrm{mol} / \mathrm{L})$ & $91.0 \pm 48.4$ & $176.0 \pm 156.5$ & $<0.001$ & $96.6 \pm 46.7$ & $90.1 \pm 35.1$ & 0.552 \\
\hline \multicolumn{7}{|l|}{ Aortic dissection features } \\
\hline \multicolumn{7}{|l|}{ Blood supply of left renal artery (\%) } \\
\hline True lumen & $326 \square 64.2 \square$ & $38 \llbracket 62.3 \square$ & & $14 \square 58.3 \square$ & $6 \square 37.5 \square$ & \\
\hline False lumen & $133 \square 26.2 \square$ & $17 \square 27.9 \square$ & & $9 \square 37.5 \square$ & $7 \square 43.8 \square$ & \\
\hline True lumen and false lumen & $49 \llbracket 9.6 \square$ & $6 \square 9.8 \square$ & 0.978 & $1 \square 4.2 \square$ & $3 \backsim 18.8 \square$ & 0.272 \\
\hline \multicolumn{7}{|l|}{ Blood supply of right renal artery (\%) } \\
\hline True lumen & $390 \square 76.8 \square$ & $41 \square 67.2 \square$ & & $18 \square 75.0 \square$ & $11 \square 68.8 \square$ & \\
\hline False lumen & $91 \square 17.9 \square$ & $13 \square 21.3 \square$ & & $4 \llbracket 16.7 \square$ & $2 \square 12.5 \square$ & \\
\hline True lumen and false lumen & $27 \square 5.3 \square$ & $7 \square 11.5 \square$ & 0.106 & $2 \llbracket 8.3 \square$ & $3 \llbracket 18.8 \square$ & 0.766 \\
\hline
\end{tabular}

Notes: Data presented as n (\%); mean \pm standard deviation.

Abbreviations: BMI, body mass index; BUN, blood urea nitrogen; sCr, serum creatinine;

Table 2 Operative data and outcome before and after propensity score matching

\begin{tabular}{|c|c|c|c|c|c|c|}
\hline \multirow[b]{2}{*}{ Characteristics } & \multicolumn{3}{|c|}{ Before Matching } & \multicolumn{3}{|c|}{ After Matching } \\
\hline & Control $(n=601)$ & CRRT $(n=111)$ & $P$ Value & Control $(n=29)$ & CRRT(n=29) & $P$ Value \\
\hline \multicolumn{7}{|l|}{ Operative procedures } \\
\hline Total arch replacement (\%) & $309(51.4)$ & $62(55.9)$ & 0.389 & $16(55.2)$ & $20(69.0)$ & 0.279 \\
\hline CABG/MVR/MVP/TVP (\%) & $51(8.5)$ & $17(15.3)$ & 0.024 & $5(17.2)$ & $4(13.8)$ & 1.000 \\
\hline Aortic valve (\%) & $185(30.8)$ & $31(27.9)$ & 0.548 & $9(31.0)$ & $7(24.1)$ & 0.557 \\
\hline \multicolumn{7}{|l|}{ CPB-related profiles } \\
\hline CPB time (min) & $235.5 \pm 64.1$ & $273.4 \pm 80.6$ & $<0.001$ & $236.0 \pm 48.8$ & $279.3 \pm 59.8$ & 0.004 \\
\hline Extracorporeal circulation assist time (min) & $54.3 \pm 31.0$ & $65.9 \pm 40.4$ & 0.001 & $50.3 \pm 30.7$ & $60.4 \pm 24.5$ & 0.174 \\
\hline Aortic cross-clamp time (min) & $165.3 \pm 55.1$ & $186.0 \pm 70.5$ & 0.005 & $174.6 \pm 48.2$ & $191.8 \pm 50.9$ & 0.192 \\
\hline DHCA time (min) & $28.0 \pm 12.7$ & $30.2 \pm 50.1$ & 0.117 & $26.8 \pm 10.4$ & $31.3 \pm 13.9$ & 0.173 \\
\hline \multicolumn{7}{|l|}{ Complications and short-time outcomes } \\
\hline Drainage volume 24 hours after surgery (ml) & $695.0 \pm 604.1$ & $1091.0 \pm 1013.9$ & 0.001 & $971.6 \pm 1104.1$ & $1011.5 \pm 681.9$ & 0.873 \\
\hline Ventilation time (hour) & $32.0 \pm 50.1$ & $56.8 \pm 61.8$ & 0.009 & $37.6 \pm 41.6$ & $79.9 \pm 81.9$ & 0.058 \\
\hline 30-day mortality (\%) & $40(6.7)$ & $50(45.0)$ & $<0.001$ & $3(10.3)$ & $13(44.8)$ & 0.003 \\
\hline ICU Stay time (day) & $6.5 \pm 11.5$ & $9.4 \pm 6.6$ & 0.020 & $5.9 \pm 5.7$ & $9.3 \pm 6.9$ & 0.055 \\
\hline Hospital stay time (day) & $22.3 \pm 11.6$ & $22.5 \pm 17.0$ & 0.892 & $19.4 \pm 10.0$ & $21.3 \pm 14.3$ & 0.560 \\
\hline
\end{tabular}

:ented as $\mathrm{n}(\%)$; mean \pm standard deviation.

:ABG, coronary artery bypass graft; MVR, mitral valve replacement; 
logistic regression analysis of Risk Factors for the groups with and without Dialysis

\begin{tabular}{lccc}
\hline & Odds Ratio & $95 \%$ CI & $P$ value \\
\hline & 1.052 & $0.984-1.052$ & 0.312 \\
& 2.027 & $0.645-6.376$ & 0.227 \\
nary artery disease & 0.319 & $0.021-4.866$ & 0.412 \\
usion & 0.552 & $0.043-7.051$ & 0.647 \\
& 1.041 & $0.966-1.123$ & 0.293 \\
TVP/TVP & 1.008 & $1.002-1.014$ & $\mathbf{0 . 0 0 5}$ \\
& 0.501 & $0.110-2.279$ & 0.371 \\
l circulation assist time & 1.022 & $1.003-1.042$ & $\mathbf{0 . 0 2 6}$ \\
lamp time & 0.997 & $0.980-1.014$ & 0.721 \\
me 24 hours after surgery & 1.000 & $1.000-1.001$ & 0.314 \\
ne & 1.006 & $0.998-1.014$ & 0.131 \\
\hline
\end{tabular}

CI, confidence interval; BUN, blood urea nitrogen; CABG, coronary artery bypass graft;

alve replacement; MVP, mitral valvuloplasty; TVP, tricuspid valvuloplasty;

lmonary bypass.

\section{Discussion}

To the best of our knowledge, the study we report herein is the first one to investigate the risk factors and outcomes of emergent surgery for TA-AAD in postoperative CRRT patients. In this study, 111 patients (15.6\%) suffered severe AKI after surgery and needed CRRT. The 30-day and long-term mortality rates were significantly higher in the CRRT group $(P<0.001)$. Our findings showed that in patients undergoing surgery for TA-AAD under the DHCA procedure, preoperative $\mathrm{SCr}$ and $\mathrm{CPB}$ time are independent risk factors for postoperative CRRT. To balance the selection bias and other confounders, baseline characteristics were matched for propensity scores. After matched, CPB time and 30-day mortality still showed significant differences between the two groups. Our Kaplan-Meier plots also revealed that the mortality of postoperative dialysis patients was significantly higher than that of the control group.

Consistent to our findings, Rice, R.D et al. [7] confirmed that the proportion of CRRT for such patients was 18.2\%. In another study conducted by Estrera et al., 347 patients with typical type A aortic dissection were analyzed, 64 cases of which suffered from CRRT (18.4\%) [6]. The incidence of postoperative CRRT in our cohort was comparable with these previous studies. CRRT procedure involved in the context of renal failure may lead to circulation instability, infection, thrombosis, and electrolyte imbalance, which will impair the postoperative recovery of patients, and even cause death [11], implying a low survival rate of 
the CRRT group as shown in our study. Elahi et al. suggested that early initiation of CRRT may reduce mortality and mortality in patients with severe AKI after cardiac surgery [12].

Elevated sCr levels before surgery was identified as a risk factor for CRRT in our study, which is consistent with previous reports $[1,13,14]$. Preoperative creatinine serum levels showed a significant influence on postoperative renal function. Elevated $\mathrm{sCr}$ levels represented damaged renal function. It is believed that increased $(>50 \%) \mathrm{sCr}$ resulted from hypotension, nephrotoxins and inflammation may lead to renal function injury [15]. Hypotension may result from cardiac tamponade or dehydration in TA-AAD patients. Reduction of renal blood flow, whether generalized or localized, plays a critical role in the occurrence of AKI [16]. Nephrotoxins include drugs and contrasts, which directly make damage to the kidney. Inflammatory response plays a major role in the development of acute aortic dissection and may also contribute to the vascular and renal parenchyma stress response, leading to the occurrence of AKI. Therapeutic strategies aimed at reducing kidney damage and accelerating function recovery contain nonpharmacologic, pharmacologic, and dialytic approaches [17]. Therefore, any therapy that can treat hypotension, nephrotoxins and inflammation regarding the increase of $\mathrm{s} \mathrm{Cr}$ may be helpful to improve the outcome, such as fluid, colloid administration for hemodynamic optimization. We'd better pay more attention when dealing with such cases because we confirmed the relationship between elevated preoperative sCr level and postoperative CRRT in our study.

Besides the sCr level, the CPB time was identified another risk factor for CRRT, as reported by Wu and colleagues [14]. Renal hypotension and hypoperfusion during CPB may be the main causes [18]. With longer duration of $\mathrm{CPB}$, more severe hypotensive periods may occur frequently. This entails prolonged period in which the mean arterial pressure is below the optimal renal autoregulation threshold [19], which may aggravate the inflammatory response and induce ischemic kidney damage [20]. Besides, circulation is stopped during aortic dissection surgery, and ischemia-reperfusion injury may occur during the operation, ischemia-reperfusion injury may lead to massive apoptosis of renal endothelial cells [21]. In addition, CPB procedure causes damage to red blood cells, and the resulting cell debris forms tiny emboli that block the renal tubular vascular network, resulting in a decrease in the filtration area of renal tubules $[22,23]$. The released hemoglobin also acts as an endogenous toxin through the release of iron, especially in the presence of low ferritin level [24]. Whether patients recover without sequela or develop AKI by progressing into the "extension phase" of kidney injury largely depends on the severity of the ensuing inflammatory response, renal hypoxia, and oxidative stress [25]. All these factors become more important if CPB is prolonged. In patients at high risk of postoperative CRRT, reduced CPB duration should be considered in perioperative care.

Based on our findings, the mortality rate of the CRRT group is significantly higher than that of the control group on 30-day and long term postoperative outcomes. A report from Japan also found that in patients who underwent surgery for TA-AAD, the mortality and major adverse cardiovascular and cerebrovascular events were correlated significantly with the severity of AKI. Furthermore, multivariate analysis showed that AKI stage 3 was an independent risk factor for mortality in those patients [26]. Sasabuchi et al [2] also reported that stage $3 \mathrm{AKI}$ defined by the KDIGO criteria had a significantly lower survival rate after 
long-term follow-up, with a median survival time of 58 months. A meta-analysis of cohort studies found that patients with AKI had higher risk of experiencing chronic kidney disease and end-stage renal disease, and the risk increased with the severity of AKI [27]. Patients with chronic kidney disease are also reported to have an increased risk of cardiovascular mortality, which contributed to the poor prognosis [28]. In addition to blood pressure control and regular assessment of aortic size, which aims to prevent aortic rupture, education for smoking cessation and weight control, using medications to lower cholesterol and to control serum blood glucose level may improve long-term survival in these patients [29].

\section{Study limitations}

Like any retrospective study of patients with TA-AAD, this study has some limitations. First of all, this is a single-center retrospective study, and there may be other confounding factors that influence the results. Secondly, despite propensity score matching was used, further potential bias and con-founders in the context of patient selection and treatment cannot be completely excluded, and the statistical power was limited due to a small patient cohort (29 pairs). Finally, our surgical technique has evolved over the study period, and our findings may have been influenced by the involvement of different surgeons.

\section{Conclusions}

In conclusion, patients receiving CRRT treatment who underwent emergency surgery for TA-AAD had higher rates of perioperative mortality and postoperative morbidity. The preoperative serum creatinine and CPB time were independent risk factors for postoperative CRRT. Further prospective multicenter studies are needed to assess the prognostic significance of CRRT and establish the most effective strategies to prevent and treat postoperative CRRT and thereby improve patient outcomes.

\section{Abbreviations}

CRRT: Continuous renal replacement therapy; TA-AAD: Type A acute aortic dissection; AKI: Acute kidney injury; sCr: Serum creatinine; CABG: Coronary artery bypass grafting; CPB: Cardiopulmonary bypass; ICU: Intensive care unit; ARF: Acute renal failure; CRF: Chronic renal failure; BMI: Body mass index; DHCA: Deep hypothermic circulatory arrest; BUN: Blood urea nitrogen; KDIGO: Kidney Disease Improving Global Outcomes; CVVH: Continuous veno-venous hemofiltration; MVR: Mitral valve replacement; MVP: Mitral valvuloplasty; TVP: Tricuspid valvuloplasty; Cl: Confidence interval

\section{Declarations}

Acknowledgements

Not applicable.

Funding 
Not applicable.

Availability of data and materials

The datasets used or analyzed during the current study are available from the corresponding author on reasonable request.

Authors' contributions

DJW, MG, ZGW designed the study; ZGW, TC, CC collected the data; ZGW, LCL analyzed the data; ZGW, QYZ analyzed and interpreted the results; DJW support and encourage the study; ZGW wrote this article; All the authors have read and reviewed this manuscript.

Ethics approval and consent to participate

The study protocol was approved by the ethics committee at Nanjing Drum Tower Hospital, and all experimental methods were performed in accordance with the relevant guidelines and regulations.

Consent for publication

Consent was obtained from the patients or their relatives.

Competing interests

The authors have declared that no interest.

\section{References}

1. Roh GU, Lee JW, Nam SB, Lee J, Choi JR, Shim YH. Incidence and risk factors of acute kidney injury after thoracic aortic surgery for acute dissection. Ann Thorac Surg. 2012;94(3):766-71. Epub 2012/06/26. doi: 10.1016/j.athoracsur.2012.04.057. PubMed PMID: 22727320.

2. Sasabuchi Y, Kimura N, Shiotsuka J, Komuro T, Mouri H, Ohnuma T, et al. Long-Term Survival in Patients With Acute Kidney Injury After Acute Type A Aortic Dissection Repair. Ann Thorac Surg. 2016;102(6):2003-9. Epub 2016/07/04. doi: 10.1016/j.athoracsur.2016.05.006. PubMed PMID: 27372373.

3. Wang J, Yu W, Zhai G, Liu N, Sun L, Zhu J. Independent risk factors for postoperative AKI and the impact of the AKI on 30-day postoperative outcomes in patients with type A acute aortic dissection: an updated meta-analysis and meta-regression. J Thorac Dis. 2018;10(5):2590-8. Epub 2018/07/13. doi: 10.21037/jtd.2018.05.47. PubMed PMID: 29997920; PubMed Central PMCID: PMCPMC6006120.

4. Hoogmoed RC, Patel HJ, Kim KM, Williams DM, Deeb GM, Yang B. Acute Kidney Injury in Acute Type B Aortic Dissection: Outcomes Over 20 Years. Ann Thorac Surg. 2019;107(2):486-92. Epub 2018/09/25. doi: 10.1016/j.athoracsur.2018.07.054. PubMed PMID: 30248320. 
5. Hobson CE, Yavas S, Segal MS, Schold JD, Tribble CG, Layon AJ, et al. Acute kidney injury is associated with increased long-term mortality after cardiothoracic surgery. Circulation. 2009;119(18):2444-53. Epub 2009/04/29. doi: 10.1161/CIRCULATIONAHA.108.800011. PubMed PMID: 19398670.

6. Estrera AL, Sandhu HK, Leake SS, Charlton-Ouw KM, Afifi RO, Miller CC, 3rd, et al. Early and late outcomes of acute type A aortic dissection with intramural hematoma. J Thorac Cardiovasc Surg. 2015;149(1):137-42. Epub 2014/12/03. doi: 10.1016/j.jtcvs.2014.10.028. PubMed PMID: 25454915.

7. Rice RD, Sandhu HK, Leake SS, Afifi RO, Azizzadeh A, Charlton-Ouw KM, et al. Is Total Arch Replacement Associated With Worse Outcomes During Repair of Acute Type A Aortic Dissection? Ann Thorac Surg. 2015;100(6):2159-65; discussion 65-6. Epub 2015/08/15. doi: 10.1016/j.athoracsur.2015.06.007. PubMed PMID: 26271582.

8. Nadim MK, Forni LG, Bihorac A, Hobson C, Koyner JL, Shaw A, et al. Cardiac and Vascular SurgeryAssociated Acute Kidney Injury: The 20th International Consensus Conference of the ADQI (Acute Disease Quality Initiative) Group. J Am Heart Assoc. 2018;7(11). Epub 2018/06/03. doi: 10.1161/JAHA.118.008834. PubMed PMID: 29858368; PubMed Central PMCID: PMCPMC6015369.

9. Kidney Disease: Improving Global Outcomes(KDIGO) Acute Kidney Injury Work Group. KDIGO Clinical Practice Guideline for Acute Kidney Injury. Kidney inter. 2012;2 (Suppl):1-138.

10. Meersch M, Schmidt C, Hoffmeier A, Van Aken H, Wempe C, Gerss J, et al. Prevention of cardiac surgery-associated AKI by implementing the KDIGO guidelines in high risk patients identified by biomarkers: the PrevAKI randomized controlled trial. Intensive Care Med. 2017;43(11):1551-61. Epub 2017/01/23. doi: 10.1007/s00134-016-4670-3. PubMed PMID: 28110412; PubMed Central PMCID: PMCPMC5633630.

11. Hanafusa N, Nakai S, Iseki K, Tsubakihara Y. Japanese society for dialysis therapy renal data registry-a window through which we can view the details of Japanese dialysis population. Kidney Int Suppl (2011). 2015;5(1):15-22. Epub 2015/06/23. doi: 10.1038/kisup.2015.5. PubMed PMID: 26097781 ; PubMed Central PMCID: PMCPMC4455188.

12. Elahi M, Asopa S, Pflueger A, Hakim N, Matata B. Acute kidney injury following cardiac surgery: impact of early versus late haemofiltration on morbidity and mortality. Eur J Cardiothorac Surg. 2009;35(5):854-63. Epub 2009/02/14. doi: 10.1016/j.ejcts.2008.12.019. PubMed PMID: 19216088.

13. Elhmidi Y, Bleiziffer S, Piazza N, Hutter A, Opitz A, Hettich I, et al. Incidence and predictors of acute kidney injury in patients undergoing transcatheter aortic valve implantation. Am Heart J. 2011;161(4):735-9. Epub 2011/04/09. doi: 10.1016/j.ahj.2011.01.009. PubMed PMID: 21473973.

14. Wu HB, Ma WG, Zhao HL, Zheng J, Li JR, Liu O, et al. Risk factors for continuous renal replacement therapy after surgical repair of type A aortic dissection. J Thorac Dis. 2017;9(4):1126-32. Epub 2017/05/20. doi: 10.21037/jtd.2017.03.128. PubMed PMID: 28523169; PubMed Central PMCID: PMCPMC5418294.

15. Brown JR, Cochran RP, Dacey LJ, Ross CS, Kunzelman KS, Dunton RF, et al. Perioperative increases in serum creatinine are predictive of increased 90-day mortality after coronary artery bypass graft 
surgery. Circulation. 2006;114(1 Suppl):I409-13. Epub 2006/07/06. doi:

10.1161/CIRCULATIONAHA.105.000596. PubMed PMID: 16820609.

16. Bonventre JV, Yang L. Cellular pathophysiology of ischemic acute kidney injury. J Clin Invest. 2011;121(11):4210-21. Epub 2011/11/03. doi: 10.1172/JCl45161. PubMed PMID: 22045571; PubMed Central PMCID: PMCPMC3204829.

17. Bellomo R, Auriemma S, Fabbri A, D'Onofrio A, Katz N, McCullough PA, et al. The pathophysiology of cardiac surgery-associated acute kidney injury (CSA-AKI). Int J Artif Organs. 2008;31(2):166-78. Epub 2008/03/04. doi: 10.1177/039139880803100210. PubMed PMID: 18311733.

18. Brezis M, Rosen S. Hypoxia of the renal medulla-its implications for disease. N Engl J Med. 1995;332(10):647-55. Epub 1995/03/09. doi: 10.1056/NEJM199503093321006. PubMed PMID: 7845430.

19. Arora P, Rajagopalam S, Ranjan R, Kolli H, Singh M, Venuto R, et al. Preoperative use of angiotensinconverting enzyme inhibitors/angiotensin receptor blockers is associated with increased risk for acute kidney injury after cardiovascular surgery. Clin J Am Soc Nephrol. 2008;3(5):1266-73. Epub 2008/08/01. doi: 10.2215/CJN.05271107. PubMed PMID: 18667735; PubMed Central PMCID: PMCPMC2518804.

20. Rosner MH, Okusa MD. Acute kidney injury associated with cardiac surgery. Clin J Am Soc Nephrol. 2006;1(1):19-32. Epub 2007/08/21. doi: 10.2215/CJN.00240605. PubMed PMID: 17699187.

21. Baines CP. The mitochondrial permeability transition pore and ischemia-reperfusion injury. Basic Res Cardiol. 2009;104(2):181-8. Epub 2009/02/27. doi: 10.1007/s00395-009-0004-8. PubMed PMID: 19242640; PubMed Central PMCID: PMCPMC2671061.

22. Valeri CR, MacGregor H, Ragno G, Healey N, Fonger J, Khuri SF. Effects of centrifugal and roller pumps on survival of autologous red cells in cardiopulmonary bypass surgery. Perfusion. 2006;21(5):291-6. Epub 2007/01/05. doi: 10.1177/0267659106073976. PubMed PMID: 17201084.

23. Moussavian MR, Slotta JE, Kollmar O, Menger MD, Schilling MK, Gronow G. Hemoglobin induces cytotoxic damage of glycine-preserved renal tubules. Transpl Int. 2007;20(10):884-94. Epub 2007/09/15. doi: 10.1111/j.1432-2277.2007.00538.x. PubMed PMID: 17854446.

24. Davis CL, Kausz AT, Zager RA, Kharasch ED, Cochran RP. Acute renal failure after cardiopulmonary bypass in related to decreased serum ferritin levels. J Am Soc Nephrol. 1999;10(11):2396-402. Epub 1999/11/30. PubMed PMID: 10541300.

25. Ho J, Lucy M, Krokhin O, Hayglass K, Pascoe E, Darroch G, et al. Mass spectrometry-based proteomic analysis of urine in acute kidney injury following cardiopulmonary bypass: a nested case-control study. Am J Kidney Dis. 2009;53(4):584-95. Epub 2008/12/17. doi: 10.1053/j.ajkd.2008.10.037. PubMed PMID: 19070948.

26. Ko T, Higashitani M, Sato A, Uemura Y, Norimatsu T, Mahara K, et al. Impact of Acute Kidney Injury on Early to Long-Term Outcomes in Patients Who Underwent Surgery for Type A Acute Aortic Dissection. Am J Cardiol. 2015;116(3):463-8. Epub 2015/06/01. doi: 10.1016/j.amjcard.2015.04.043. PubMed PMID: 26026862. 
27. Coca SG, Singanamala S, Parikh CR. Chronic kidney disease after acute kidney injury: a systematic review and meta-analysis. Kidney Int. 2012;81(5):442-8. Epub 2011/11/25. doi: 10.1038/ki.2011.379. PubMed PMID: 22113526; PubMed Central PMCID: PMCPMC3788581.

28. Chronic Kidney Disease Prognosis C, Matsushita K, van der Velde M, Astor BC, Woodward M, Levey AS, et al. Association of estimated glomerular filtration rate and albuminuria with all-cause and cardiovascular mortality in general population cohorts: a collaborative meta-analysis. Lancet. 2010;375(9731):2073-81. Epub 2010/05/21. doi: 10.1016/S0140-6736(10)60674-5. PubMed PMID: 20483451; PubMed Central PMCID: PMCPMC3993088.

29. Patel SA, Winkel M, Ali MK, Narayan KM, Mehta NK. Cardiovascular mortality associated with 5 leading risk factors: national and state preventable fractions estimated from survey data. Ann Intern Med. 2015;163(4):245-53. Epub 2015/06/30. doi: 10.7326/M14-1753. PubMed PMID: 26121190.

\section{Figures}

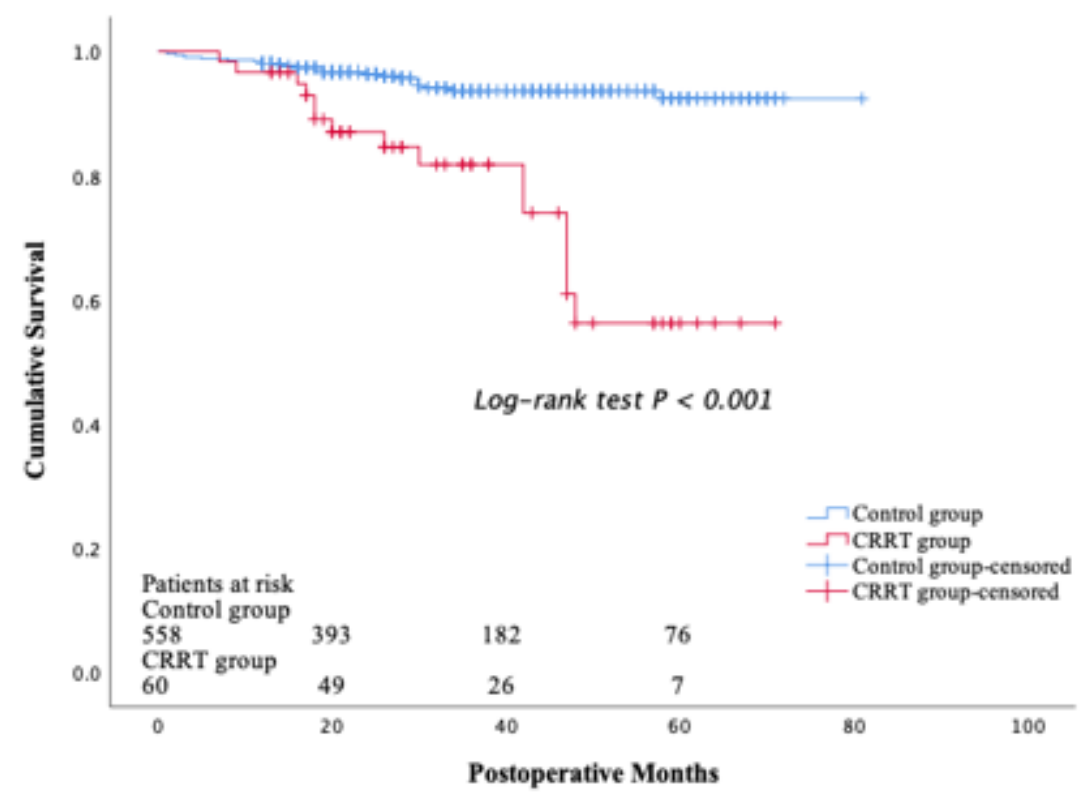

\section{Figure 1}

Long-term overall cumulative survival after surgical aortic repair for type A acute aortic dissection in CRRT and control groups with significant different risk profiles: Kaplan-Meier estimation. Patients were censored at the cut-off of the study. 